\title{
A criticism of the reductionist and holistic vision in the planning of drugs in biological, chemical and physical level
}

\author{
Daniel Augusto Barra de Oliveira ${ }^{1+}{ }^{\oplus}$, Edenilson dos Santos Niculau ${ }^{1}$
}

1. Federal University of Tocantins, Integrated Sciences Center, Araguaína, Brazil.

+Corresponding author: Daniel Augusto Barra de Oliveira, Phone: +55 (63) 99200-7791, Email address: danielchem@uft.edu.br

\section{ARTICLE INFO}

Article history:

Received: June 12, 2020

Accepted: May 19, 2021

Published: July 01, 2021

Section Editor: Assis Vicente Benedetti
Keywords

1. drug design

2. reductionism

3. holism

4. organicism

5. systems biology

ABSTRACT: In this work, two philosophical premises of science applied in the understanding of diseases and in the planning of drugs were studied. The first premise is reductionism. This idea is present in modern science when a problem can be reduced to the sum of its individual parts. Diseases can be understood as the metabolic action of few enzymes. Drugs can be planned through the mimicry of a specific enzymatic substrate. Biological molecules can be explained by the quantum theory applied to atoms and molecules. This idea has been the predominant way in modern science. On the other hand, there is a holistic view of the phenomenon. In this holistic view, the phenomenon must be understood as the whole. Drug design should be thought from a network of proteins, not just from a single enzymatic target. There is in fact a slight advantage in the reductionist method, because this philosophical view simplifies the problem. Today, a holistic view combined with methodological reductionism is used to develop new potential drugs.
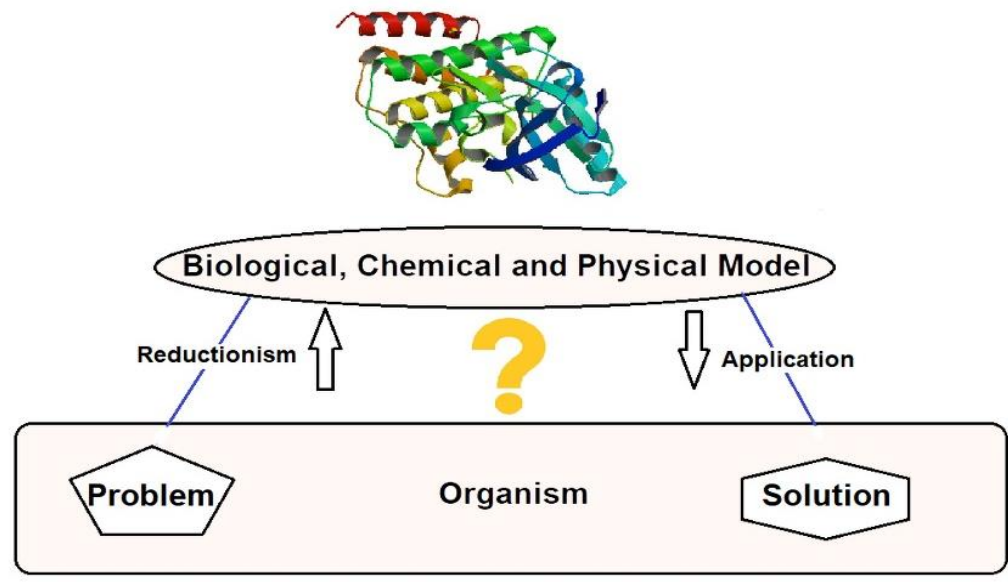

\section{CONTENTS}

1. Introduction

2. Methodology

3. Drug design

4. The origin of biological reductionism

5. Chemical reductionism

6. Physical reductionism
7. The problem in design of new drugs

8. Organicism as a counterproposal in cancer treatment

8.1 Systems biology

8.2 Predicting drug-target interactions (DTI)

9. Concluding remarks 


\section{Introduction}

Discussion between reductionist and holistic methods have been used to understand the nature of matter. Democritus and Leucippus, the "creators" of the atom idea, were the first to establish a reductionist explanation of matter assuming the existence of atoms. On the other hand, Aristotle admitted holistic explanations. There are three types of reductionism. Ontological reductionism is justified on the assumption that behavior of the whole can be explained by the sum of the individual properties of the components. Methodological reductionism is based on the idea that scientific explanation must be reduced to the smallest elements. The explanation of a particular phenomenon can be made in terms of the most fundamental constituents of this phenomenon. The methodological reductionism is very clear in some scientific discussion above molecular drug planning, because several biochemical processes are described by molecular and atom association. Researchers have employed quantum mechanics to describe biochemical phenomena. The fundamental constituents of matter are used to develop new drugs. Lastly, there is the epistemological reductionism, which a theory takes the form of a deductive argument where the premises are the primary theory and the conclusion is the secondary theory. The theories of biology can be derived as special cases of the laws and theories of the physical sciences (Zalta et al., 2017). Holistic view contrasts with the reductionist premise by assuming that the explanation of the phenomenon must necessarily describe the whole. In this case, the interactive aspects of the individual components are considered. In this paper, the reductionist and holistic assumptions are discussed to comprehend diseases and drug design. This discussion is interesting because it can be used to trace the scientific pathways to be used in the study of a particular scientific phenomenon.

Nowadays, the properties of atoms can be obtained from the quantum mechanics equations. The fundamental particles, that are explained by quantum mechanics, can be used to describe the chemical properties of atoms and molecules. The biomolecules that are the components of life can be explained through physical and chemical phenomena. Finally, biology becomes nothing more than a cluster of atoms that explain the phenomenon of life as a whole. This idea was advocated by Schrödinger (1946). The reductionism gains a new perspective by quantum theory. Individual molecules can be used to explain diseases as a whole. Defective genes may be the cause of cancer. A drug can be designed due to the idea that a specific enzyme interacts with a biological receptor. This molecular interaction controls a complex biological response. Currently, reductionism is the theory most employed to understand the existence of diseases and design new drugs for treating illness.

In opposition to reductionism, the holistic vision assumes that diseases must be comprehended by individual components interactions. In order to develop new drugs, the researcher must study the biological system as a whole. A molecule does not interact with a single enzyme because it can have a different effect from what was planned. Biochemical mechanisms should be understood based on the interactions between the biomolecules as a whole and not as a summation of individual properties. Thus, it is important that the researcher gives special attention to these visions in order to find a way to understand the biological mechanisms and rules that govern life.

In this manuscript, the discussion of reductionism is performed at the biological, chemical, and physical levels to understand the development of drugs and diseases. After the previous discussion about reductionism paradigms applied to drug discovery, the holistic idea is discussed in order to understand the same problems using the biology of systems. Methodological reductionism and holism are not entirely opposite to each other. Each approach has limitations.

\section{Methodology}

The purpose of this manuscript is to show how ontological reductionism brings difficulties to understand diseases and design new drugs. Holistic approach derived from the biology of the system is presented in the manuscript. This discussion extends at a biological, chemical, and physical level. The old and current literature were also evaluated to show the two premises adopted in the study and development of drugs. Finally, methodological reductionism is used to build holistic approaches, showing the complementarity of both approaches.

\section{Drug design}

The discussion on the use of proteins in drug design began more than 35 years ago with the emergence of information on the three-dimensional (3D) structure of globin and polypeptide hormones (Schechter, 2008). The protein structures are used as biological targets, virtual screening and fragment screening. 
The development of structural genomics provided more 3D structures that can be determined by gene sequences. These methodologies were particularly important for the development of computational methods, which can help to identify the sites involved in the intermolecular interaction with the inhibitors.

First, it is possible to use silicon approaches (virtual screening and redesign) to select a subset of samples from a large compound file. A new drug can also be designed from previously identified ligands. Secondly, molecular modeling can be used to study the interaction between a possible biological target and the protein receptor. The evaluation of the activity of a possible drug can be made by the similarity to the original substrate, through the interactions performed by the possible drug, or using a multilinear regression to perform a study of structure and activity relationship. Molecular modeling uses molecular structure and electronic calculations to study conformational changes and molecular interactions between an enzyme and a potential drug candidate. The electronic structure calculations are based on methodologies derivative from quantum mechanics approximations. Now, there is prior information (docking, quantum chemistry simulations, Quantitative structure-activity relationship [QSAR]) for a rational organic synthesis of the new drug. The synthesized drug will be tested in vitro and in vivo. The success of these tests may or may not lead to a new drug. Briefly, rational drug design is preceded by the choice of a biological target. This biological target can be known or created by sequencing the amino acids. The elucidation of molecular structure will allow the comprehension of the interaction between the biological target with the substrates. Subsequently, the electronic structure and molecular mechanical calculations will be used to understand the enzymatic active site. Molecular docking is used to generate a set of conformations inside the catalytic site. The comprehension about the catalytic site will identify which amino acids interact with the possible drug. In this stage, the theoretical approaches will help to establish the most important hydrogen bonds, pi stacking interactions, ion interaction and induced dipole interactions. The bind energy can be calculated using classical force fields or quantum mechanics/molecular mechanics (QM/MM) approaches.

Next step is to start the synthesis of the drug candidate. Then, this molecule may be assayed on in vitro enzymes, for example. This is a brief summary of the well-known "rational" design of new drugs. In the manuscript, it will be shown that the idea behind the rational design of drugs starts from ontological reductionism, which does not cover the whole problem to be solved.

\section{The origin of biological reductionism}

The discussion about reductionism in different meanings can be observed in the declarations below.

Crick (1966) claims that "The ultimate aim of the modern movement in biology is to explain all biology in terms of physics and chemistry". This approach epitomizes the reductionist mindset that has permeated molecular biology for half a century. The most extreme manifestation of the reductionist view is the belief that is held by some neuroscientists that consciousness and mental states can be reduced to chemical reactions that occur in the brain.

The epistemological reductionism can be observed in the words of Crick (1966). The domain of biology is reduced to chemical reactions. It could be possible to predict biological phenomena due to specific chemical reactions. On the other hand, Paul Nurse criticized the reductionism defended by Crick and other scientists.

Nurse (1997) begins his writing in nature with a reductionist question:

"If we had knowledge of all the molecular reactions that take place within the cell, would we know the cell?" The article comes up with the following answer: "Explanations in science must always have some elements of reductionism, but descriptions of increasing detail may only provide a delusion of understanding; overenthusiastic pursuit of reductionism can limit discovery and also has ideological implications in defining what is considered to be the best science in terms of publication and financial support..." However, there is a real problem to define what is an enough explanation.

There are many philosophers who believe that there is a reduction from causality at the macro level to causality at the micro level. Menzies (1988) calls this idea causal reductionism. Reducing causal relationships from macro to micro levels presupposes some way to correlate the events that have causation effect at different levels.

Ontological reductionism analyzes the whole in parts and decides the associations between the parts. This approach accepts that only the molecules supply a comprehension of the entire system.

Currently, it is evident that the specificity of a complex biological activity does not emerge from the properties of the individual molecules. This molecular 
structure constantly works in numerous distinctive forms (Nicholson, 2019; Pierce Junior et al., 1960). There are yet studies that lead to a holistic view for the treatment of certain types of disease (Birkbak and McGranahan, 2020; Nicholson, 2019; Pierce Junior et al., 1960). The cancer treatment is a particularly interesting case. Cancer is a disease of multifactorial origin that is treated in the context of that the problem originated from somatic mutations in cells. Barry Pierce and his colleagues showed the differentiation of malignant neoplastic cells into benign cell types (Lok, 2006; Pierce Junior et al., 1960). These researchers refuted the initial dogma "once a cancer cell, always a cancer cell". The microenvironment and normal tissue architectures may restrict tumor development, but otherwise may also promote and induce cancer (Hagios et al., 1998; NCI, 2018). A brief survey on the page of the National Cancer Institute in the United States (Manley et al., 2002) shows that many of the drugs employed for cancer treatment assume that the problem is restricted to cellular communication. A methodological reductionism will be applied to develop drugs against cancer. Many of the drugs are inhibitors of the enzyme tyrosine kinase (Jemal et al., 2010; Shah et al., 2020). The treatment of a disease begins with the initial idea that the interaction of a molecule with an enzyme will give responses for a multifactorial disease. There is a clear limitation of the complexity of the problem. This limitation has an approach based on ontological reductionism (when the disease is based on the idea that the constituents can explain the whole) at the same time will apply methodological reductionism to understand the disease (due to technological limitations, a simple model is chosen to start the study about the disease).

Until 1990, mortality rates had expanded a lot. After the $90 \mathrm{~s}$, a gradual decrease in the number of cases was observed, mainly due to cancer prevention through tobacco control and other healthy behaviors (Chabner and Roberts Junior, 2005). However, patients with strong tumors do not react to any drugs (Chabner and Roberts Junior, 2005; Ecker, 2015). In any case, the persistence of cancer stem cells and unfavorable medicate impacts still restrain their capacity to stabilize or cure malignant invasion in the long run (Cardoso, 2020; Chabner and Roberts Junior, 2005; Ecker, 2015). The mortality rates had a modest decrease in recent years, but the cancer remains a major cause of death within the industrialized world. The reasons are complex: insufficient tumor models used within the different cancer screening programs; critical long-term harmfulness of anticancer drugs; reaction rates in patients due to sedate affectability; rapid evolution of aggressive drug resistant cells due to high mutation rates and selective pressure, resulting in transient treatment responses (Johnson, 2013; Lok, 2006; Ponting and Russell, 2002; Zhong and Virshup, 2020).

Methodological reductionism approaches to biomedical experiments have provided significant insight into the predominant regions associated with specific functions. Such discoveries, in turn, led to significant applications. For example, antibody fragments such as antigen binding fragment (Fab), single chain variable fragment $(\mathrm{scFv})$ or fragment chain $(\mathrm{Fc})$ are widely used as screening reagents and as therapeutic potentials (Ahn et al., 2006; Van Regenmortel, 2004). Structural refinement protein domain classifications and functional predictions are additionally used for therapeutic purposes (Krakauer et al., 2017). However, the reductionist approach, while accessible and incredibly useful, ignores the broader framework of interregional communications and their possible cooperative effects (Krakauer et al., 2017) that would be useful for further analysis. Given the advances in technology that have led to advanced experimental and computational techniques in recent years, the subsequent level of scientific advances may require proteins to be analyzed in the most holistic way. These approaches are already present in various specialties with these efforts to jointly generate ideas derived from reductionist investigation (Albergante et al., 2016; Bieber, 2015; Fardet, 2014; Phua et al., 2019). According to Van Regenmortel (2004), it is important to revisit biological systems completely as a whole (O'Sullivan et al., 2018; Soto and Sonnenschein, 2005). In this line of thinking, while limitations on looking at whole systems are always present, it is possible, however, to already be reaching a saturation point for scientific advances within the reductionist approach. Recent literature has suggested an analysis of proteins in their entirety (when possible) (van Ommen et al., 2008).

The reductionist nutritional approach thrived using the tools of analytical chemistry and experimental biology in nutrition. Recent advances in high-yield organic molecules complex structure (OMIC) technology, computational and statistical tools have led scientists to explore current challenges. These tools provide global measurements reporting the diversity of individuals and complex interactions between vitamins and human bodies (Davis and Hord, 2005; Omenn et al., 1996; Weinstock, 2012). High-performance omics technology includes genomics, transcriptomics, proteomics, metabolomics and metagenomics. Nutrigenomics, or genomics in nutrition, refer to the study of how genes and dietary components interact to 
change the phenotype (Blumberg, 1994; ChiritaEmandi and Niculescu, 2020). The three genomic categories in which knowledge is critically necessary are human genomics, plant and animal genomics, and microbial genomics (Davis and Hord, 2005). Nutrigenomics explain how the response to food components depends on an individual's genetic background (nutrigenetics), nutrient-induced changes in DNA methylation, chromatin changes (nutritional epigenetics), nutrient-induced changes in gene expression (nutritional transcriptomics) and proteins (nutritional proteomics) (Blumberg, 1994). Metabolomics is one of the newest omics and has been defined as a comprehensive analysis of changes in many low molecular weight compounds and their fluxes through human metabolism in response to dietary treatments (Davis and Hord, 2005).

Metagenomics studies the combined genome of microbial communities using next generation of DNA sequencing, which explains differences in community structure between sampling sites, individuals, and between healthy versus disease states (Rietjens et al., 2002). A good example of limitation related to the application of reductionism in nutritional research can be found in prospective studies that investigate the effects of the intake of isolated antioxidant vitamin intake and cancer development (Fardet and Rock, 2014; Peterson, 2008). The results showed controversies. Although antioxidants become prooxidants after exerting their antioxidant effect in in vitro systems, pro-oxidant formation is rapidly attenuated by recyclable chain reactions involving glutathione in the human body (Fojo, 2008). However, at a very high dose, antioxidant vitamins can lead to toxic pro-oxidant actions, indicating the absence of linear cause and effect association.

The vitamins may not have the same activity in crude and characteristic foods (Fardet and Rock, 2014). There is a demand that the potential for nourishment depends on both supplement composition and nourishment structure properties, frequently driving to conflicting discoveries. Subsequently, it was expected that a more comprehensive vision of antioxidant potential would approach a few different angles of cancer prevention agents with approaches based on synergistic, adversarial, or multi-component and multitarget additives.

Examples of reductionism at the biological level and its implications are common. Here, diseases and health problems are reduced to biological molecules, such as DNA, RNA, and specific enzymes. It is necessary to know biological targets for the synthesis of new drugs. There is yet a technological obstacle that prevents the study of the system as a whole. In this way, methodological reductionism must be comprehended as a step to reach ontological holism. However, a new type of reductionism is noted when scientists reduce the problem to simple molecules.

\section{Chemical reductionism}

Modern chemistry was born with Lavoisier's quantitative studies. On the other hand, biology has been developed since classical antiquity. Aristotle had manuscripts classifying the living beings. Nowadays there is a particular branch or knowledge shared by chemistry and biology. These sciences use methodologies derivative from biochemistry. The studies in both disciplines start by creating rigorous tests to study molecules, genes or proteins. However, the targets are remarkably diverse in both disciplines. Biological systems use the comparison between two systems to understand connections, uncovering tall arrangements in organized structures and modeling complexity. On the other hand, biochemistry is ruled by the attitude of matching pairing individual compounds and molecular targets. As a result, efficient biochemistry ponders are frequently centered on query databases to recognize interactions between particular molecules and a single target.

In general, the salicylic acid is described as a cyclooxygenase (COX) (Fig. 1) inhibitor, atorvastatin as a 3-hydroxy-3-methylglutaryl (HMG) coenzyme A reductase inhibitor and lithium as a glycogen synthase kinase $3 \beta$ (GSK3 $\beta$ ) inhibitor.

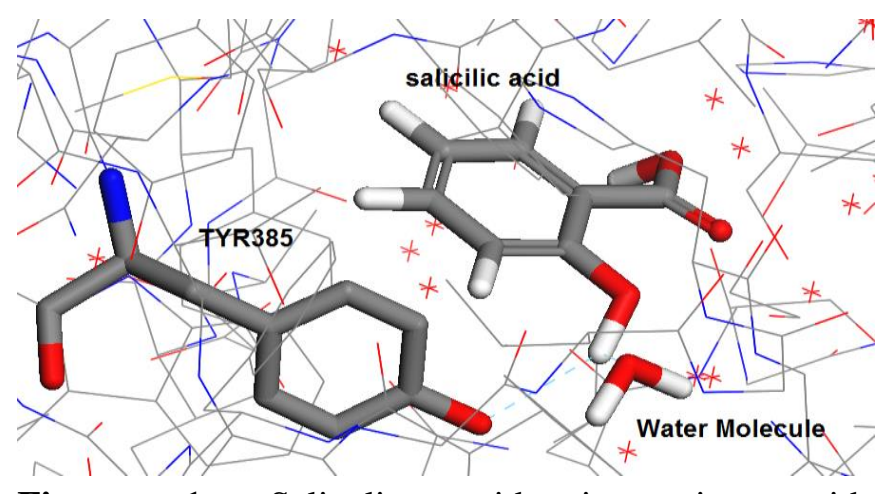

Figure 1. Salicylic acid interacting with cyclooxygenase site. Structure obtained from 5F1A (The Crystal Structure of Salicylate Bound to Human Cyclooxygenase-2/Protein Data Bank).

These descriptors are useful and carry some information, but, in reality, these small molecules do much more than inhibit a single enzyme. The benefits and negative effects on humans are not fully explained 
by their actions on a single enzyme. It is important to invoke a holism view of the system. Selective kinase inhibitors in clinical oncology were the main goal of research a decade ago. However, many experts now believe that kinase inhibitors may be advantageous in treating cancer due to the multi target reached by these kinds of molecules. However, the rational drug design continues to follow the idea that "Cell life is closely linked to a large number of specific and selective interactions between bio macromolecules" (Cheng et al., 2012; Cavasotto et al., 2018). Macromolecular modeling by docking studies provides drug-receptor interaction. In this approach, the drug structure is designed based on its 3D adjustment to the receptor site structure (Aucar and Cavasotto, 2020). A clear example is the inhibitors of enzyme kinase. Kinases are enzymes associated with the biochemical process known as signal transduction. In this process, the enzyme kinase catalyzes phosphorylation reactions of other enzymes. This biochemical process triggers a cascade of chemical signals that are converted into physical responses by the cell. In this case, the target protein is chosen for the first step of rational drug design. At this stage, the drug is designed in order to mimic the structure of the adenosine triphosphate (ATP) molecule. The premise is that the drug must have ATP-common pharmacophoric groups in order to inhibit enzyme activity through the induced docking model proposed by Koshland (1958). In this model, the substrate is able to adapt to changes in enzyme conformation. This idea permeates the construction of new drugs aimed at cancer treatment (Akhtar et al., 2019; Müller et al., 2019). However, these molecules act in different enzyme targets. Dasatinib is an example of a drug that interacts with several kinases. Dasatinib is a targeted therapy medication used to treat certain cases of chronic myeloid leukemia and Philadelphia chromosome-positive acute lymphoblastic leukemia. Dasatinib is a potent bioavailable oral inhibitor of several kinases, including breakpoint cluster region protein in ABL genes (BCR-ABL) (Fig. 2), protooncogene tyrosine-protein kinase SRC, c-KIT and platelet-derived growth factor receptor beta (PDGFR$\beta$ ) (Lombardo et al., 2004). It has been discovered by synthesizing and testing a series of thiazole-based compounds with activity against SRC and ABL kinases (Lombardo et al., 2004).

However, the dasatinib interaction is not limited to kinases. The in vivo pharmacokinetic study shows that pretreatment with dasatinib monohydrate decreased the blood level of CsA (cyclosporine) in rats, perhaps due to induction of cytochrome P450 3A4 (CYP3A2) isoenzymes (Abdelgalil et al., 2019). The cycle- oxygenase inhibitor aspirin also acts on different enzyme targets. Aspirin blocks the formation of metastatic intravascular niches by inhibition of plateletderived COX-1/thromboxane A2 (Ramasarma, 1994). The intrinsic properties of a protein allow catalysis of many reactions.

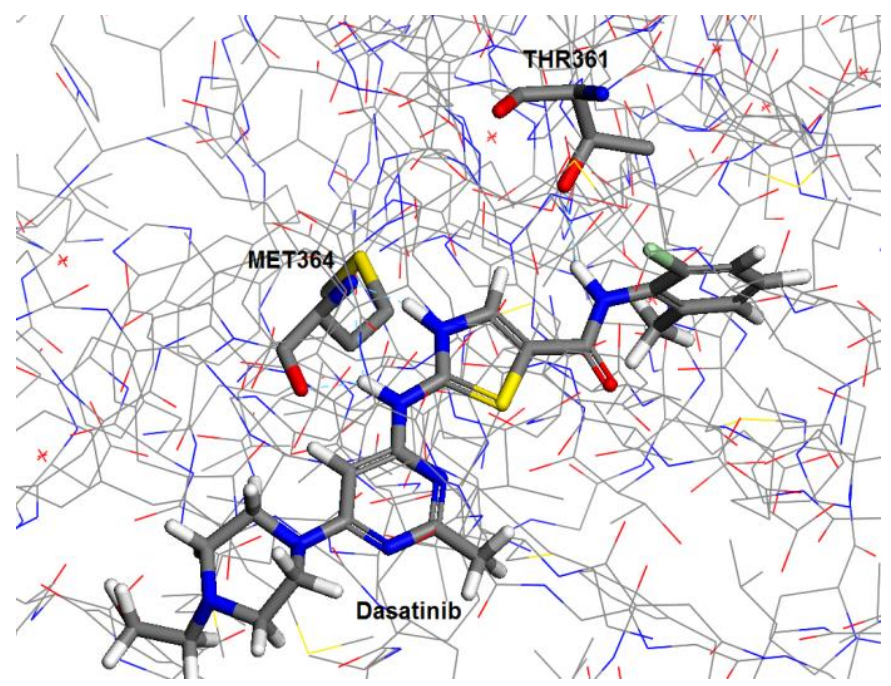

Figure 2. Dasatinib interacting with ABL2/ARG kinase. Crystal structure of ABL2/ARG kinase in complex with dasatinib obtained from protein data bank code 4 XLI.

Ramasarma (1994) describes a list of more than fifty proteins with the capacity to know several kinds of molecules. These elective capacities incorporate a set of activities. These biomolecules act as enzymes, particle carriers and inhibit different cellular processes. It was discovered that intensive information of the physical and chemical properties of a protein will not give data about what it does. The question about what chemistry can contribute to the whole biological process is not so clear. Reductionism would explain which proteins produce effects on the biological system. This idea was created by Pigliucci and Kaplan (2010). It is exceptionally common to say that a protein is an esterase, kinase, etc., which appears as its primary function. The protein glyceraldehyde 3-phosphate dehydrogenase (GAPDH) plays an important role in glyceraldehyde 3-phosphate catalysis. Glyceraldehyde 3-phosphate dehydrogenase is a bio molecule with a specific function. This enzyme does not require a specific molecular structure, but an environment in which glyceraldehyde-3-phosphate is changed. Chemistry alone cannot tell that a specific protein is GAPDH. This bio molecule will only perform the specific activity if it has an environment that 
incorporates other components that together make its work particular.

While chemistry concerns molecular structures, biochemistry will work with these particles inside a system. The exclusive study about the molecule will not offer a complete comprehension about the function of biomolecules (Alm and Arkin, 2003). Genes that influence natural product fly memory arrangement encode cyclic adenosine monophosphate (cAMP) proteins within the signaling pathway are not a particular memory. It is the cell compartment and the environment in which a cAMP is discharged that permits a hereditary item to have an impact. Natural specificity comes from the way that these components gather and work together.

\section{Physical reductionism}

The early twentieth century had a significant transformation in the context of atomic structure. The old Greeks tried to explain the existence of atoms from philosophical central conjectures. Dalton later differentiated atoms with the help of the balance. However, the atoms remained nondivisible, as that of the Greeks (Democritus and Leucippus). Thomson used the cathode ray tube to discover electrons. Rutherford bombardments a gold plate to discover the existence of the nucleus. Planck begins quantum mechanics studying the heat from materials at high temperatures. Einstein theorizes the corpuscular nature using the photoelectric effect. Schrödinger (1926) developed a wave equation whose solutions are the quantum numbers. At this point, the reductionism of chemistry to physics began, as Dirac (1929) points out:

"The general theory of quantum mechanics is now almost complete, the imperfections that still remain being in connection with the exact fitting in of the theory with relativity ideas. These give rise to difficulties only when high-speed particles are involved, and are therefore of no importance in the consideration of atomic and molecular structure and ordinary chemical reactions, in which it is, indeed, usually sufficiently accurate if one neglects relativity variation of mass with velocity and assumes only Coulomb forces between the various electrons and atomic nuclei. The underlying physical laws necessary for the mathematical theory of a large part of physics and the whole of chemistry are thus completely known, and the difficulty is only that the exact application of these laws leads to equations much too complicated to be soluble."

This is a clear epistemological reductionism defended by Dirac. It is not only a methodological approach, but a complete explanation of chemistry using the quantum mechanics equations. However, it will be shown that the presumptions made by Dirac are not entirely correct. Although a methodological reductionism can be used to simulate the atoms and molecules.

Physical-level reductionism is visible in biomolecule modeling for new drug planning purposes. The behavior of biological molecules is studied from quantum approaches, semi-empirical and classical models. In this case, the molecules and their properties are explained by quantum mechanics and their approximations. In general, the coordinates of atoms are given. From these coordinates, atomic number, mass, among other properties, are extracted chemical and biological properties of interest. The heart of quantum theory for atoms and molecules is the resolution of the Schrodinger equation. However, this equation has no analytical solution for atoms with more than one electron. Approaches are required to solve this problem. One of the first approximations to facilitate the resolution of the Schrödinger equation is the Born-Oppenheimer approximation. In this approach, it is assumed that the motion of the atomic nucleus and electrons can be separated (Born and Oppenheimer, 1927). Chemical structure cannot be found in pure quantum mechanics applied to a chemical system. It is imposed by the BornOppenheimer approach (Woolley, 1978). New approaches were developed to explain the chemical bond originating quantum chemistry methodologies

The introduction of the electron spin and the Pauli exclusion principle were used to explain the formation of a chemical bond. The linear combination of atomic orbitals (LCAO) and valency theory were used to describe the chemical bond. However, there is the problem associated with the Schrodinger equation solution to many electrons. Several approaches were used to solve this limitation. The first, already presented, is that of Born-Oppenheimer. The other approximations are dependent on the method used. It is important to note that neither approach solves the Schrödinger equation analytically. Hartree's (1928a, b) approach, for example, considers the interaction of an electron with the average field generated by the other electrons. Jordan and Fock (1930) introduced the antisymmetric fermion product in Hartree's method. Finally, Roothaan (1951) added the linear combination of atomic orbitals to the method. Hartree's initial approximation contains a limit in the system energy, which will always be greater than that obtained by the exact solution of the Schrödinger equation. For Woolley (1978), the concept of molecular structure is 
absent at the actual quantum level. Electronic structure calculations are based on approximations.

Löwdin (1955) denominated the difference between exact energy for that calculated from the ab-initio approach as correlation energy. The lowest and most accurate energy would be found by overcoming the Born-Oppenheimer approximation and by inclusion of relativistic corrections. Post Hartree-Fock approaches named coupled cluster use iterative single, double and triple perturbative excitations $(\operatorname{CCSD}(\mathrm{T}))$ that are able to perform calculations with a precision of $\pm 5 \mathrm{~kJ} \mathrm{~mol}^{-1}$ for small and medium molecules (Kümmel, 2002; Shavitt and Bartlett, 2009). There is still the problem with computational time. Robust calculations, such as those derived from M $\phi$ ller-Plesset perturbation theory (MP2 and MP3 methods) and those approaches that use double and triple excitations are computationally unviable for the treatment of biomolecules. The classic force fields are employed to study biological molecules. In this method, quantum mechanics is replaced by classical mechanics. Electrons are forgotten and chemical bonds are treated as springmass systems. Interactions are described by electrostatic and van der Waals equations. This method is the molecular mechanics (Kümmel, 2002; Leach, 2001) that allows the study of optimization and dynamics of proteins and other biological molecules. Systematic validations based on quantum mechanics have been performed in order to standardize the molecular force fields (van der Spoel, 2021).

Rational drug design at the molecular level is generally based on X-ray diffraction. In this technique, the position of atoms in the crystal is determined to obtain the three-dimensional structure of the biomolecule. This structure can be studied as a whole with classical mechanics. There is also the possibility of temporal evolution of the system using classical force fields associated with the integration of Newton's equations step by step in time. This technique is known as molecular dynamics. The longest molecular dynamic ever performed was on the order of $10^{-6} \mathrm{~s}$. The problem is that some biochemical mechanisms, such as protein folding, are in the temporal interstice of $10^{-3} \mathrm{~s}$. In the study of structure and activity relationship (SAR), the activity of a set of known drugs can be correlated with a series of their physicochemical properties. The idea behind this technique is to construct a multilinear regression curve that relates known drug activity to a particular universe of physicochemical properties. The graph allows the inferences of the activity of a new drug before the synthesis.
Fundamental presumption for QSAR is that comparable molecules have comparable activities. This rule is called the structure-activity relationship. The fundamental issue is how to characterize a little distinction at the molecule level, since each sort of movement and response capacity, biotransformation capacity, solvency, target movement, and so on, may depend on another distinction. Great examples have been given in the literature (Brown, 2012; Patani and LaVoie, 1996).

The speculations depend on a limited amount of chemical information. The rule of acceptance must be regarded to maintain a strategic distance from over adjusted theories and to determine over adjusted elucidations in basic molecular information. The reality is that not all comparative molecules have comparative activities.

It was shown several reductionism kinds. Dirac (1929) defends a complete epistemological reductionism, which quantum mechanics premises is the primary theory and the conclusion is chemistry. On the other hand, Crick (1966) argues that biology can be reduced to chemical reactions. Both these propositions were criticized, showing arguments against this kind of reductionism. It is important to declare that biology developed itself without the knowledge of chemistry. There is not a causal dependence between chemistry, biology and physics.

However, methodological reductionism is applied nowadays to begin the studies in drug design. It will show the problems associated with methodological reductionism. The holistic approaches that can be used to work with drug design will be described as an alternative to approaches merely reductionist.

\section{The problem in design of new drugs}

The number of modern drugs approved by Food and Drug Administration (FDA) diminished over the last years. The number of approved drugs reduced from 10 to 20 during the year of 2002. This decline has been held in spite of proceeded industry mergers, acquisitions and yearly investing over US\$ 30 billion. Some commentators qualify this declination due to organization causes, such as wasteful venture administration, expanded prerequisites, a decrease in clinical science that bargains with entirety living beings, an overemphasis on technology-driven inquire about, and a need of eagerness to do so, center on items that are not anticipated to create deals of at slightest US\$ 0.5 to 1.0 billion per year (Drews, 2003; Fojo, 2008; Gershell and Atkins, 2003; Kubinyi, 2003; Miska, 2003). Furthermore, these results show that 
methodologies based on tall throughput screening, combinatorial chemistry, genomics, proteomics, and bioinformatics are not bringing the modern conquests that were predicted (Glassman and Sun, 2004; Kubinyi, 2003; Miska, 2003).

Information about human genome arrangements and different pathogens led to a set number of modern drug targets (Drews, 2003). In addition, a few biotechnology enterprises fizzled to correspond to the perspectives in order to establish quality treatment, investigating antisense innovation and cancer immunizations. A common issue with numerous of these advancements is that the potential for dangers and undesirable side impacts tend to be ignored, as was the case with gene therapy (Glassman and Sun, 2004).

However, there is a probable reason for these disappointments: most of these approaches have been guided by supreme reductionism. As a result, the complexity of organic frameworks, whole life forms and patients tends to be underestimated (Horrobin, 2001). Illnesses results from the interaction of numerous genes and sometimes it is conceivable to know all the genes and hereditary included in a specific biological function.

Another field of biomedical science strongly influenced by reductionist theory is the so-called vaccine design, which is based on the premise that the concepts of drug design based on molecular structure can be applied to vaccines (Van Regenmortel, 2001; 2021). However, this approach neglects that the relationship between a drug and its receptor or molecular target is exceptionally particular, whereas the relationship between an antigen and a counter acting agent is much less limited. The binding site of an immunoglobulin molecule comprises approximately 50 hypervariable residues that together constitute the complementarity determining regions (CDRs). Hypervariable residues together make the CDRs. In general, around $10-15$ of these residues have an interest in interaction with a specific epitope, but the total complement of all 50 hypervariable residues does not constitute a real binding site for any epitope. This implies that approximately 35 of the CDRs residues can possibly bind to other epitopes that are small or no likeness to the previous. This complexity clarifies the broad multi-specificity of immunoglobulins and the numerous distinctive paratopes or bind sites on each molecule. The capacity of an immunoglobulin molecule to bind to different antigenic structures is improved by the impressive adaptability of CDRs. The molecule of immunoglobulin has different conformations with several binding sites (Bosshard, 2001; Goh et al., 2004; James et al., 2003). In in the 2000s, rational drug design has become a necessity in the advancement of antibody research contradicting observational data (Van Regenmortel, 2000). The term rational drug design suggests that this investigation uses molecular information and structural knowledge, whereas the term design shows that the developed products are predictable. Rational drug design is considered the more logical approach than experimental "trial-and-error" screening and molecular selection.

The conviction that a molecular design plan will be effective for the improvement of unused antibodies is ordinary of the reductionist approach. Ontological reductionism expects that a biological phenomenon can be decreased to the chemistry level. However, there are numerous reasons that show that reductionist approaches to antibody advancement are impossible to succeed. First, the antigenic determinants or epitopes of an infectious agent are rising substances that are characterized by their particular antibody partners and exist as it were within the setting of the immune system. Epitopes and paratopes are not intrinsic characteristics of an antigen. Immunoglobulin individually cannot be recognized autonomously by a binding reaction. Second, the idea behind the antibodies that essentially bind to the pathogen have small consideration in immunization advancement. Antibodies that have a functional activity are required; specifically, the capacity to neutralize the infectious agents in vivo. Human capacity to anticipate protein function is constrained. The ability to predict neutralizing action of an antibody by chemical structure is for all intents and purposes inexistent (Van Regenmortel, 2000; 2002). Immunization and protective resistance have meaning at the level of the entire living being: particles, tissues and organs cannot be vaccinated. Immunization takes place in the organic domain and cannot be decreased to the level of chemistry. Third, in spite of an exceptional worldwide request for investigation efforts using reductionist approaches, no immunodeficiency infection antibody is in sight (Burton and Moore, 1998). Reductionist approach on HIV immunization advancement continues to be advocated (Burton et al., 2004), although there is no evidence that it will be efficient. This approach includes deciding the atomic structure of monoclonal antibodies to HIV antigens using X-ray crystallography to illustrate the structure of HIV epitopes. The basis for these studies is the suspicion that information of the structure of epitopes that are recognized by neutralizing antibodies will offer assistance to plan a viable antibody against HIV. Crystallographic X-ray investigation of HIV- 
neutralizing antibodies may describe the structure of epitopes inside the molecular pockets, but it does not tell how to utilize immunization and initiate antibodies with the same specificity (Van Regenmortel, 2002; Burton and Moore, 1998). The structures of epitopes and paratopes that are displayed in a complex describe the last conformation of an energetic handle by the alteration in the somatic change. It is not conceivable that the conformation of the epitope on the immunogen is eventually responsible for the appearance of neutralizing antibodies.

\section{Organicism as a counterproposal in cancer treatment}

The progress in molecular science over the past three decades has cleared the way for a hereditary cancer hypothesis, the somatic mutation theory (SMT) (Boveri, 1929; Weinberg, 1998). This hypothesis qualifies as a "standard hypothesis" because it has collected most of the financing for cancer investigation. This hypothesis assumes that cancer was generated from genetic breakdown at the cellular level.

This model of cancer can be followed back to the work of Boveri (1929). Nowadays, it is ordinarily defended by Weinberg (1998). This reductionist approach was explicitly exposed in Weinberg's book One Renegade Cell, that emphasizes that the basic part of a single mutated cell will originate from cancer (Weinberg, 1998). One alternative comes from traditional biology and emphasizes that the living being must be studied as a whole. The central point of this research is the work of Waddington (1935). According to the tissue organizational field theory (TOFT), the causes of cancer should be investigated not at the hereditary level, but at the tissue level. The TOFT claims that cancer starts from a disturbance of tissue organization. This theory has been examined by Dolberg and Bissell (1984) and characterizes the conceptual system with an organicist point of view.

The SMT approach, whereby the cause of cancer must be inquired about at the hereditary level, has regularly been qualified as an illustration of reductionism, and more accurately as hereditary reductionism. The ideas behind SMT inside hereditary qualities based on reductionist paradigm really rose within the 1970s. At the time, an impressive number of carcinogenic chemicals could cause hereditary changes.

Afterward, it was found that a few of the so-called tumor infections (also called quality transformers or oncogenes) could lead to the advancement of tumors that carry hereditary transformations in tainted cells. At this point, the hereditary cause of cancer changed from exogenous to endogenous and a few endogenous oncogenes were distinguished as transformed shapes of ordinary cell qualities.

Later researches showed the true complexity of cancer. It was found that a few cases of carcinogenesis could be understood as a multistep to prepare numerous oncogenes, as well as possibly one or more anti-oncogenes or tumor silencers. Around 100 oncogenes and 15 anti-oncogenes have been recognized (Hanahan and Weinberg, 2000). The cell cycle clock was considered critical, as were the characteristics of the cell life. The explanation described by Weinberg has been amplified beyond intracellular hereditary causes. Nowadays, there are numerous biomolecular pathways, including signals of communication between cells. In carcinogenesis, the cells acquire six particular capacities: specification, "self-sufficiency" in development signals, coldheartedness inhibitory development signals (antigrowth), avoidance of modified cell passing (apoptosis), replicative potential boundless, maintained angiogenesis in tissue (Hanahan and Weinberg, 2000).

Each of these capabilities may be obtained by distinctive molecular pathways: self-sufficiency in development signals coming about in independent cell development and multiplication that may be searched by the modification of extracellular development signals, transcellular transducers of such signals or circuits, which decipher these signals into activity. These six capabilities would indeed be empowered by a seventh characteristic, the genomic precariousness, in some cases it is known as "more prominent variability". The definition of the SMT has been reformulated in terms of "heterotypic intuitive" between early tumor cells and their ordinary neighbors. (Hanahan and Weinberg, 2000). Hereditary reductionism, which describes the origin of cancer by a cell mutation, has been adjusted to cover the complexity of carcinogenesis and the variety of atomic pathways that lead to tumor cell multiplication. Although SMT presents a clear limitation to explain the cancer origin, the paradigm centered on molecular substances remains the most fundamental explanation.

Cancer approach proposed by Sonnenschein and Soto (2020) and their group is based on tissue investigation. The TOFT finds an explanation of cancer in terms of disturbance of tissue organization as opposition to the expression of a defective gene. The TOFT is based on two fundamental presumptions: (i) expansion is the default state of all cells, and (ii) carcinogens act initially due to distribution of the 
interaction that happens between cells within the stroma and parenchyma of an organ.

For TOFT researchers, the SMT program works with a reductionist problem, because it looks for carcinogenesis caused at the genes and molecular components level of cells instead of the total level of tissues: cancer is seen as an intracellular problem caused by mutations within the DNA of the cancer cell. The basic level that carcinogenesis causes ought to be explored is the tissue level, because this level is used in biopsy that can give an authoritative conclusion of cancer (Soto and Sonnenschein, 2005). Sonnenschein and Soto (2020) characterize hereditary reductionism as: a great number of biologists demand that cancer clarifications should be analyzed in gene or gene product level. This reductionist-genetic predicts that everything in biology can be diminished to genes since the genome is the store of transmissible information.

The approach taken by SMT is exceptionally prohibitive because it limits the issue in terms of genes and molecular expressions. It has indeed condemned the cancer investigation. For this reason, this theory should be eliminated or supplanted. In other words, the SMT approach is criticized to reduce the cancer to genes and particles inside cells. This approach will inhibit the exam of other potential causes, such as tissue organization. As a substitute for hereditary reductionism, TOFT proposes an approach based on organicism: cancer is seen as an issue comparable to histogenesis or organogenesis and is hence a developmental science. Organicists "select to work" at the level of organization at which the phenomenon is observed. The TOFT cautiously wanders into lower levels of organization, slowly moving through the different various levels in which the phenomenon is observed.

\subsection{Systems biology}

The objective of systems biology is to consider the physiological and conditions of administrative arrangement levels, signaling pathways, cells, tissue, organs, and inevitably the whole life form (Berg, 2014). It is a holistic view based in some methodological reductionism to compute the properties that will integrate the whole. The structure of science comprises a number of approaches and models that help within the consideration of the organic complexity of different illnesses. System biology combines an expansive sum of reductionist genomic information, proteomic and metabolomic tests to produce an organized data set for considering a disease.
System biology points to the complex behavior of organic frameworks that develop from personal framework components and intelligence between them (Sobie et al., 2011). Hence, a fundamental highlight of system biology is that the interaction between all framework components is examined instead of the characteristics of each individual component. It gives a few approaches to creating forecasts that can be tested experimentally. System biology depends on the combination of test ponders, that creates information concerning cellular components of a framework as well as approaches that help within the examination of different information sets. Two major computational approaches are utilized in system biology, specifically, data oriented (top-down approach) and hypothesis driven (bottom-up approach) (Faratian et al., 20093) to create new treatments.

\subsection{Predicting drug-target interactions (DTI)}

Drugs often bind to more than one biomolecular target. It is fundamental to get the polypharmacology of a medicament. Exploratory approaches to identify DTI are costly, difficult, and time expending. Hence, computational approaches are broadly used for DTI expectation. Computational approaches characterize each target by a set of known connection covers, searching for chemically organized drugs anticipating modern DTIs (Keiser et al. 2009). Associations between drugs and targets can be anticipated based on the chemical structure of the drug and protein sequence (Li et al., 2015; 2017). Drug-target interactions can moreover be distinguished based on similitudes of side impacts, i.e., drugs with comparable side impacts tend to be associated with the same biological targets (Berg, 2014; Campillos et al., 2008). The combination of pharmacogenomic likenesses and side impacts are used in DTI (Li et al., 2017). In addition, a comparison of three administered induction strategies to anticipate DTI is the induction likeness, target-based likeness deduction, and network-b.

Complex diseases are controlled by the interconnected systems of numerous pathways related to cell expansion, attack and medicate resistance (Ryall and Tan, 2015). In this way, it is troublesome to create modern treatments against complex infections. Drugtarget interactions approach uses a combination of medicating treatments that at the same time balance numerous targets and may have more advantages than employing a single drug (Jia et al., 2009). This approach gives strong evidence for anticipating treatments of an infection. A few network-based approaches have been employed to anticipate drug 
combinations for cancer treatment. In this calculation the kinase inhibition profile and the region with tumor are used to develop a set of drugs as well as to anticipate tumor sensitivities for new drugs or drug combinations ( $\mathrm{Pal}$ and Berlow, 2012). Vital kinases for mesenchymal and epithelial cell migration were anticipated, employing a joint approach to strong cancer drug combinations. This pooled approach uses flexible net regularization with mRNA expression profiling and a huge set of kinase inhibition.

\section{Concluding remarks}

Today, there is a clear application of methodological reductionism to develop new drugs although there is a grasp that holism must be reached to understand the full biological process. On the other hand, holistic approaches like system biology use some reductionist methodologies to build a whole vision of the organism. Nowadays, a balancing of methodological reductionist approach and the holistic view is combined to produce new drugs and understand new diseases.

\section{Authors' contribution}

Conceptualization: Augusto, D. A. B.

Data curation: Not applicable.

Formal Analysis: Not applicable.

Funding acquisition: Not applicable.

Investigation: Augusto, D. A. B.; Niculau, E. S.

Methodology: Not applicable.

Project administration: Not applicable.

Resources: Not applicable.

Software: Not applicable.

Supervision: Not applicable.

Validation: Not applicable.

Visualization: Not applicable.

Writing - original draft: Not applicable.

Writing - review \& editing: Augusto, D. A. B.; Niculau, E. S.

\section{Data availability statement}

Data sharing is not applicable.

\section{Funding}

Not applicable.

\section{Acknowledgments}

Not applicable.

\section{References}

Abdelgalil, A. A.; Alam, M. A.; Raish, M.; Mohammed, I. E.; Mohammed, A.-E. H.; Ansari, M. A.; Al Jenoobi, F. I. Dasatinib significantly reduced in vivo exposure to cyclosporine in a rat model: The possible involvement of CYP3A induction. Pharmacol. Rep. 2019, 71 (2), 201-205. https://doi.org/10.1016/j.pharep.2018.10.018.

Ahn, A. C.; Tewari, M.; Poon, C.-S.; Phillips, R. S. The limits of reductionism in medicine: could systems biology offer an alternative? PLoS Med. 2006, 3 (6), e208. https://doi.org/10.1371/journal.pmed.0030208.

Akhtar, N.; Jabeen, I.; Jalal, N.; Antilla, J. Structure-based pharmacophore models to probe anticancer activity of inhibitors of protein kinase B-beta (PKB $\beta)$. Chem. Biol. Drug. Des. 2019, 93 (3), 325-336. https://doi.org/10.1111/cbdd.13418.

Albergante, L.; Liu, D.; Palmer, S.; Newman, T. J. Insights into Biological Complexity from Simple Foundations. In Biophysics of Infection: Advances in Experimental Medicine and Biology; Springer, 2016; pp 295-305. https://doi.org/10.1007/978-3-319-32189-9_18.

Alm, E.; Arkin, A. P. Biological networks. Curr. Opin. Struct. Biol. 2003, $13 \quad$ (2), 193-202. https://doi.org/10.1016/S0959-440X(03)00031-9.

Aucar, M. G.; Cavasotto, C. N. Molecular Docking Using Quantum Mechanical-Based Methods. In Quantum Mechanics in Drug Discovery; Humana, 2020. https://doi.org/10.1007/978-1-0716-0282-9_17.

Berg, E. L. Systems biology in drug discovery and development. Drug Discov. Today 2014, 19 (2), 113-125. https://doi.org/10.1016/j.drudis.2013.10.003.

Bieber, T. Concept and Scientific Background of Personalized Medicine. In Personalized Treatment Options in Dermatology; Springer, 2015; pp 1-5. https://doi.org/10.1007/978-3-662-45840-2_1.

Birkbak, N. J.; McGranahan, N. Cancer Genome Evolutionary Trajectories in Metastasis. Cancer Cell 2020, 37 (1), 8-19. https://doi.org/10.1016/j.ccell.2019.12.004.

Blumberg, J. The Alpha-Tocopherol, Beta-Carotene Cancer Prevention Study in Finland. Nutr. Rev. 1994, 52 (7), 242 245. https://doi.org/10.1111/j.1753-4887.1994.tb01430.x. 
Born, M.; Oppenheimer, R. Zur Quantentheorie der Molekeln. Ann. Phys. (Berl.) 1927, 389 (20), 457-484. https://doi.org/10.1002/andp.19273892002.

Bosshard, H. R. Molecular Recognition by Induced Fit: How Fit is the Concept? News Physiol. Sci. 2001, 16, 171-173. https://doi.org/10.1152/physiologyonline.2001.16.4.171.

Boveri, T. The Origin of Malignant Tumors; Baillière, Tindall \& Cox, 1929.

Brown, N. Bioisosterism in Medicinal Chemistry. In Bioisosteres in Medicinal Chemistry; Wiley-VCH, 2012; pp 1-14. https://doi.org/10.1002/9783527654307.ch1.

Burton, D. R.; Desrosiers, R. C.; Doms, R. W.; Koff, W. C.; Kwong, P. D.; Moore, J. P.; Nabel, G. J.; Sodroski, J.; Wilson, I. A.; Wyatt, R. T. HIV vaccine design and the neutralizing antibody problem. Nat. Immunol. 2004, 5, 233236. https://doi.org/10.1038/ni0304-233.

Burton, D. R.; Moore, J. P. Why do we not have an HIV vaccine and how can we make one? Nat. Med. 1998, 4, 495498. https://doi.org/10.1038/nm0598supp-495.

Campillos, M.; Kuhn, M.; Gavin, A.-C.; Jensen, L. J.; Bork, P. Drug target identification using side-effect similarity. Science 2008, $321 \quad$ (5886), 263-266. https://doi.org/10.1126/science.1158140.

Cardoso, E.; Guidi, M.; Blanchet, B.; Schneider, M. P.; Decosterd, L. A.; Buclin, T.; Csajka, C.; Widmer, N. Therapeutic Drug Monitoring of Targeted Anticancer Protein Kinase Inhibitors in Routine Clinical Use: A Critical Review. Ther. Drug Monit. 2020, 42 (1), 33-44. https://doi.org/10.1097/FTD.0000000000000699.

Cavasotto, C. N.; Aucar, M. G.; Adler, N. S. Computational chemistry in drug lead discovery and design. Int. J. Quantum Chem. 2018, $119 \quad$ (2), e25678. https://doi.org/10.1002/qua.25678.

Chabner, B. A.; Roberts Junior, T. G. Chemotherapy and the war on cancer. Nat. Rev. Cancer 2005, 5 (1), 65-72. https://doi.org/10.1038/nrc1529.

Cheng, F.; Liu, C.; Jiang, J.; Lu, W.; Li, W.; Liu, G.; Zhou, W.; Huang, J. Prediction of Drug-Target Interactions and Drug Repositioning via Network-Based Inference. PLoS Comput. Biol. 2012, 8 (5), e1002503. https://doi.org/10.1371/journal.pcbi.1002503.

Chirita-Emandi, A.; Niculescu, M. Methods for Global Nutrigenomics and Precision Nutrition. In Principles of Nutrigenetics and Nutrigenomics: Fundamentals for Individualized Nutrition; Academic Press, 2020; pp 49-58. https://doi.org/10.1016/B978-0-12-804572-5.00007-0.
Crick, F. Of Molecules and Men; University of Washington Press, 1966.

Davis, C. D.; Hord, N. G. Nutritional "Omics" Technologies for Elucidating the Role(s) of Bioactive Food Components in Colon Cancer Prevention. J. Nutr. 2005, 135 (11), 2694 2697. https://doi.org/10.1093/jn/135.11.2694.

Dirac, P. A. M. Quantum mechanics of many-electron systems. Proc. R. Soc. A 1929, 123 (792), 714-733. https://doi.org/10.1098/rspa.1929.0094.

Dolberg, D. S.; Bissell, M. J. Inability of Rous sarcoma virus to cause sarcomas in the avian embryo. Nature 1984, 309, 552-556. https://doi.org/10.1038/309552a0.

Drews, J. Strategic trends in the drug industry. Drug Discov. Today 2003, 8 (9), 411-420. https://doi.org/10.1016/S13596446(03)02690-4.

Ecker, D. M.; Jones, S. D.; Levine, H. L. The therapeutic monoclonal antibody market. MAbs 2015, 7 (1), 9-14. https://doi.org/10.4161/19420862.2015.989042.

Faratian, D.; Clyde, R. G.; Crawford, J. W.; Harrison, D. J. Systems pathology - taking molecular pathology into a new dimension. Nat. Rev. Clin. Oncol. 2009, 6 (8), 455-464. https://doi.org/10.1038/nrclinonc.2009.102.

Fardet, A. Food health potential is primarily due to its matrix structure, then nutrient composition: a new paradigm for food classification according to technological processes applied. J Nutr. Health Food Eng. 2014, 1 (5), 208-209. https://doi.org/10.15406/jnhfe.2014.01.00031.

Fardet, A.; Rock, E. Toward a New Philosophy of Preventive Nutrition: From a Reductionist to a Holistic Paradigm to Improve Nutritional Recommendations. Adv. Nutr. 2014, $\quad 5 \quad$ (4) 430-446. https://doi.org/10.3945/an.114.006122.

Fojo, T. Commentary: Novel Therapies for Cancer: Why Dirty Might Be Better. Oncologist 2008, 13 (3), 277-283. https://doi.org/10.1634/theoncologist.2007-0090.

Gershell, L. J.; Atkins, J. H. A brief history of novel drug discovery technologies. Nat. Rev. Drug Discov. 2003, 2, 321-327. https://doi.org/10.1038/nrd1064.

Glassman, R. H.; Sun, A. Y. Biotechnology: identifying advances from the hype. Nat. Rev. Drug Discov. 2004, 3, 177-183. https://doi.org/10.1038/nrd1309.

Goh, C.-S.; Milburn, D.; Gerstein, M. Conformational changes associated with protein-protein interactions. Curr. Opin. Struct. Biol. 2004, 14 (1), 104-109. https://doi.org/10.1016/j.sbi.2004.01.005.

Hagios, C.; Lochter, A.; Bissell, M. J. Tissue architecture: the ultimate regulator of epithelial function? Phil. Trans. $R$. 
Soc. Lond. B 1998, $353 \quad$ (1370), 857-870. https://doi.org/10.1098/rstb.1998.0250.

Hanahan, D.; Weinberg, R. A. The Hallmarks of Cancer. Cell 2000, 100 (1), 57-70. https://doi.org/10.1016/S00928674(00)81683-9.

Hartree, D. R. The Wave Mechanics of an Atom with a NonCoulomb Central Field. Part II. Some Results and Discussion. Math. Proc. Camb. Philos. Soc. 1928a, 24 (1), 111-132. https://doi.org/10.1017/S0305004100011920.

Hartree, D. R. The Wave Mechanics of an Atom with a nonCoulomb Central Field. Part III. Term Values and Intensities in Series in Optical Spectra. Math. Proc. Camb. Philos. Soc. 1928b, $\quad 24$ (3), 426-437. https://doi.org/10.1017/S0305004100015954.

Horrobin, D. F. Realism in drug discovery-could Cassandra be right? Nat. Biotechnol. 2001, 19, 1099-1100. https://doi.org/10.1038/nbt1201-1099.

James, L. C.; Roversi, P.; Tawfik, D. S. Antibody Multispecificity Mediated by Conformational Diversity. Science 2003, $299 \quad$ (5611), $1362-1367$. https://doi.org/10.1126/science.1079731.

Jemal, A.; Ward, E.; Thun, M. Declining Death Rates Reflect Progress against Cancer. PLOS ONE 2010, 5 (3), e9584. https://doi.org/10.1371/journal.pone.0009584.

Jia, J.; Zhu, F.; Ma, X.; Cao, Z., Cao, Z. W.; Li, Y. X.; Yu, Z. C. Mechanisms of drug combinations: interaction and network perspectives. Nat. Rev. Drug Discov. 2009, 8, 111128. https://doi.org/10.1038/nrd2683.

Johnson, M. Antibody Structure and Antibody Fragments. Mater. Methods 2013, $\quad 3, \quad 160$. https://doi.org/10.13070/mm.en.3.160.

Jordan, P.; Fock, V. Neue Unbestimmtheit Eigenschaften des elektromagnetischen Feldes. Z. Physik 1930, 66, 206-209. https://doi.org/10.1007/BF01392901.

Keiser, M. J.; Setola, V.; Irwin, J. J.; Laggner, C.; Abbas, A. I.; Hufeisen, S. J.; Jensen, H. N.; Kuijer, M. B.; Matos, R. C.; Tran, T. B.; Whaley, Glennon, R. A.; Hert, J.; Thomas, K. L. H.; Edwards, D. D.; Shoichet, B. K.; Roth, B. L. Predicting new molecular targets for known drugs. Nature 2009, 462, 175-181. https://doi.org/10.1038/nature08506.

Koshland, D. E. Application of a theory of enzyme specificity to protein synthesis. Proc Natl Acad Sci 1958, 44(2), 98-104. https://doi.org/10.1073/pnas.44.2.98.

Krakauer, J. W.; Ghazanfar, A. A.; Gomez-Marin, A.; Maclver, M. A.; Poeppel, D. Neuroscience Needs Behavior: Correcting a Reductionist Bias. Neuron 2017, 93 (3), 480490. https://doi.org/10.1016/j.neuron.2016.12.041.
Kubinyi, H. Drug research: myths, hype and reality. Nat. Rev. Drug Discov. 2003, 2, 665-668. https://doi.org/10.1038/nrd1156.

Kümmel, H. G. A Biography of the coupled cluster method. In: Recent Progress in Many-Body Theories; World Scientific, 2002; pp 334-348. https://doi.org/10.1142/9789812777843_0040.

Leach, A. R. Molecular Modelling: Principles and Applications; Pearson Education, 2001.

Li, P.; Huang, C.; Fu, Y.; Wang, J.; Wu, Z.; Ru, J.; Zheng, C.; Guo, Z.; Chen, X.; Zhou, W.; Zhang, W.; Li, Y.; Chen, J.; Lu, A.; Wang, Y. Large-scale exploration and analysis of drug combinations. Bioinformatics 2015, 31 (12), 20072016. https://doi.org/10.1093/bioinformatics/btv080.

Li, Z.; Han, P.; You, Z.-H.; Li, X.; Zhang, Y.; Yu, H.; Ru, $\mathrm{N}$.; Xing, C. In silico prediction of drug-target interaction networks based on drug chemical structure and protein sequences. Sci. Rep. 2017, 7, 11174. https://doi.org/10.1038/s41598-017-10724-0.

Lok, C. Thinking outside the cell. Nat Biotechnol. 2006, 24, 111-112. https://doi.org/10.1038/nbt0106-111.

Lombardo, L. J.; Lee, F. Y.; Chen, P.; Norris, D.; Barrish, J. C.; Behnia, K.; Castaneda, S.; Cornelius, L. A. M.; Das, J.; Doweyko, A. M.; Fairchild, C.; Hunt, J. T.; Inigo, I.; Johnston, K.; Kamath, A.; Kan, D.; Klei, H.; Marathe, P.; Pang, S.; Peterson, R.; Pitt, S.; Schieven, G. L.; Schmidt, R. J.; Tokarski, J.; Wen, M.-L.; Wityak, J.; Borzilleri, R. M. Discovery of $N$-(2-Chloro-6-methyl- phenyl)-2-(6-(4-(2hydroxyethyl)- piperazin-1-yl)-2-methylpyrimidin-4ylamino)thiazole-5-carboxamide (BMS-354825), a Dual Src/Abl Kinase Inhibitor with Potent Antitumor Activity in Preclinical Assays. J. Med. Chem. 2004, 47 (27), 66586661. https://doi.org/10.1021/jm049486a.

Löwdin, P.-O. Quantum Theory of Many-Particle Systems. III. Extension of the Hartree-Fock Scheme to Include Degenerate Systems and Correlation Effects. Phys. Rev. 1955, 97 (6), 1509-1520. https://doi.org/10.1103/PhysRev.97.1509.

Manley, P. W.; Cowan-Jacob, S. W.; Buchdunger, E.; Fabbro, D.; Fendrich, G.; Furet, P.; Meyer, T.; Zimmermann, J. Imatinib: a selective tyrosine kinase inhibitor. Eur. J.

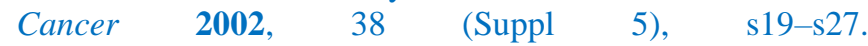
https://doi.org/10.1016/S0959-8049(02)80599-8.

Menzies, P. Against Causal Reductionism. Mind 1988, 388, 551-574. https://doi.org/10.1093/mind/XCVII.388.551.

Miska, D. Biotech's twentieth birthday blues. Nat. Rev. Drug Discov. 2003, 2, 231-233. https://doi.org/10.1038/nrd1036. 
Müller, J.; Kirschner, R. A.; Geyer, A.; Klebe, G. Conceptual Design of Self-Assembling Bisubstrate-like Inhibitors of Protein Kinase A Resulting in a Boronic Acid Glutamate Linkage. ACS Omega 2019, 4 (1), 775-784. https://doi.org/10.1021/acsomega.8b02364.

National Cancer Institute (NCI). In: Definitions. Qeios, 2018. https://www.cancer.gov/ (accessed 2020-02-21).

Nicholson, D. J. Is the cell really a machine? J. Theor. Biol. 2019, 477 , 108-26.

https://doi.org/10.1016/j.jtbi.2019.06.002.

Nurse, P. The ends of understanding. Nature 1997, 387 (6634), 657. https://doi.org/10.1038/42600.

O’Sullivan, A.; Henrick, B.; Dixon, B.; Barile, D.; Zivkovic, A.; Smilowitz, J.; Lemay, D.; Martin, W.; German, J. B.; Schaefer, S. E. 21st century toolkit for optimizing population health through precision nutrition. Crit. Rev. Food Sci. Nutr. 2018, 58 (17), 3004-3015. https://doi.org/10.1080/10408398.2017.1348335.

Omenn, G. S.; Goodman, G. E.; Thornquist, M. D.; Balmes, J.; Cullen, M. R.; Glass, A.; Keogh, J. P.; Meyskens Junior, F. L.; Valanis, B.; Williams Junior, J. M.; Barnhart, S.; Cherniack, M. G.; Brodkin, C. A.; Hammar, S. Risk Factors for Lung Cancer and for Intervention Effects in CARET, the Beta-Carotene and Retinol Efficacy Trial. J. Natl. Cancer Inst. $\quad \mathbf{1 9 9 6}, \quad 88 \quad$ (21), $1550-1559$. https://doi.org/10.1093/jnci/88.21.1550.

Pal, R.; Berlow, N. A kinase inhibition map approach for tumor sensitivity prediction and combination therapy design for targeted drugs. Pacific Symp. Biocomput. 2012, 351-362. https://doi.org/10.1142/9789814366496_0034.

Patani, G. A.; LaVoie, E. J. Bioisosterism: A Rational Approach in Drug Design. Chem. Rev. 1996, 96 (8), 31473176. https://doi.org/10.1021/cr950066q.

Peterson, R. T. Chemical biology and the limits of reductionism. Nat. Chem. Biol. 2008, 4 (11), 635-638. https://doi.org/10.1038/nchembio1108-635.

Phua, S.-X.; Chan, K.-F.; Su, C. T.-T.; Poh, J.-J.; Gan, S. K.E. Perspective: The promises of a holistic view of proteinsimpact on antibody engineering and drug discovery. Biosci. Rep. 2019, $39 \quad$ (1), $\quad$ BSR20181958. https://doi.org/10.1042/BSR20181958.

Pierce Junior, G. B.; Dixon Junior, F. J.; Verney, E. L. Teratocarcinogenic and tissue-forming potentials of the cell types comprising neoplastic embryoid bodies. Lab Invest. 1960, 9, 583-602.

Pigliucci, M.; Kaplan, J. Making Sense of Evolution: The Conceptual Foundations of Evolutionary Biology; University of Chicago Press, 2010.
Ponting, C. P.; Russell, R. R. The natural history of protein domains. Annu. Rev. Biophys. Biomol. Struct. 2002, 31, 4571.

https://doi.org/10.1146/annurev.biophys.31.082901.134314.

Ramasarma, T. One protein - Many functions. Curr. Sci. 1994, 67 (1), 24-29.

Rietjens, I. M. C. M.; Boersma, M. G.; de Haan, L.; Spenkelink, B.; Awad, H. M.; Cnubben, N. H. P.; van Zanden, J. J.; van der Woude, H.; Alink, G. M.; Koeman, J. H. The pro-oxidant chemistry of the natural antioxidants vitamin C, vitamin E, carotenoids and flavonoids. Environ. Toxicol. Pharmacol. 2002, 11 (3-4), 321-333. https://doi.org/10.1016/S1382-6689(02)00003-0.

Roothaan, C. C. J. New Developments in Molecular Orbital Theory. Rev. Mod. Phys. 1951, 23 (2), 69-89. https://doi.org/10.1103/RevModPhys.23.69.

Ryall, K. A.; Tan, A. C. Systems biology approaches for advancing the discovery of effective drug combinations. $J$. Cheminform. 2015, 7, 7. https://doi.org/10.1186/s13321-0150055-9.

Schechter, A. N. Hemoglobin research and the origins of molecular medicine. Blood 2008, 112 (10), 3927-3938. https://doi.org/10.1182/blood-2008-04-078188.

Schrödinger, E. An Undulatory Theory of the Mechanics of Atoms and Molecules. Phys. Rev. 1926, 28(6), 1049-1070. https://doi.org/10.1103/PhysRev.28.1049.

Schrödinger, E. What is Life? J. Philos. 1946, 43 (7), 194195. https://doi.org/10.2307/2019342.

Shah, P.; Dylgjeri, F.; Srinivasan, B.; Brennan, P. A. Oral tyrosine kinase inhibitors in OMFS: a review. J. Oral Maxillofac. Surg. 2020, $58 \quad$ (2), 135-138. https://doi.org/10.1016/j.bjoms.2019.11.022.

Shavitt, I.; Bartlett, R. J. Many-Body Methods in Chemistry and Physics: MBPT and Coupled-Cluster Theory; Cambridge University Press, 2009. https://doi.org/10.1017/CBO9780511596834.

Sobie, E. A.; Lee, Y.-S.; Jenkins, S. L.; Iyengar, R. Systems Biology_Biomedical Modeling. Sci. Signal 2011, 4 (190), tr2. https://doi.org/10.1126/scisignal.2001989.

Sonnenschein, C.; Soto, A. M. Over a century of cancer research: Inconvenient truths and promising leads. PLoS $\begin{array}{lllll}\text { Biol. } & \mathbf{2 0 2 0}, & 18 & \text { (4), } & \text { e3000670. }\end{array}$ https://doi.org/10.1371/journal.pbio.3000670.

Soto, A. M.; Sonnenschein, C. Emergentism as a default: Cancer as a problem of tissue organization. J. Biosci. 2005, 30 (1), 103-118. https://doi.org/10.1007/BF02705155. 
van der Spoel, D. Systematic design of biomolecular force fields. Curr. Opin. Struct. Biol. 2021, 67, 18-24. https://doi.org/10.1016/j.sbi.2020.08.006.

van Ommen, B.; Cavallieri, D.; Roche, H. M.; Klein, U. I.; Daniel, $\mathrm{H}$. The challenges for molecular nutrition research 4 : the "nutritional systems biology level". Genes Nutr. 2008, 3, 107-113. https://doi.org/10.1007/s12263-008-0090-5.

Van Regenmortel, M. H. V. Are there two distinct research strategies for developing biologically active molecules: rational design and empirical selection? J. Mol. Recognit. 2000, 13 (1), 1-4. https://doi.org/10.1002/(SICI)10991352(200001/02)13:1\%3C1::AID-JMR490\%3E3.0.CO;2-W.

Van Regenmortel, M. H. V. Pitfalls of reductionism in the design of peptide-based vaccines. Vaccine 2001, 19 (17-19), 2369-2374. https://doi.org/10.1016/S0264-410X(00)004564.

Van Regenmortel, M. H. V. Reductionism and complexity in molecular biology. Scientists now have the tools to unravel biological and overcome the limitations of reductionism. EMBO Rep. 2004, 5 (11), 1016-1020. https://doi.org/10.1038/sj.embor.7400284.

Van Regenmortel, M. H. V. Reductionism and the search for structure-function relationships in antibody molecules. $J$. Mol. Recognit. 2002, $15 \quad$ (5), 240-247. https://doi.org/10.1002/jmr.584.

Van Regenmortel, M. H. V. What does it mean to develop an HIV vaccine by rational design? Arch. Virol. 2021, 166, $27-$ 33. https://doi.org/10.1007/s00705-020-04884-0.

Waddington, C. H. Cancer and the Theory of Organisers. Nature 1935, 135, 606-608. https://doi.org/10.1038/135606a0.

Weinberg, R. A. One Renegade Cell: The Quest for the Origins of Cancer; Weidenfeld and Nicolson: Science Masters, 1998.

Weinstock, G. M. Genomic approaches to studying the human microbiota. Nature 2012, 489 (7415), 250-256. https://doi.org/10.1038/nature11553.

Woolley, R. G. Must a molecule have a shape? J. Am. Chem. Soc. $\quad 1978, \quad 100 \quad$ (4), $1073-1078$. https://doi.org/10.1021/ja00472a009.

Zalta, E. N.; Nodelman, U.; Allen, C.; Anderson. R. L. Reductionism in Biology. Stanford Encyclopedia of Philosophy, 2017.

https://plato.stanford.edu/entries/reduction-biology/

(accessed 2021-04-21).
Zhong, Z.; Virshup, D. M. Wnt Signaling and Drug Resistance in Cancer. Mol. Pharmacol. 2020, 97 (2), 72-89. https://doi.org/10.1124/mol.119.117978. 